\title{
Sedentary behaviour and physical activity are associated with biomarkers of endothelial dysfunction and low-grade inflammation—relevance for (pre)diabetes: The Maastricht Study
}

\author{
Evelien J. Vandercappellen ${ }^{1,2,3}$ (D) Annemarie Koster ${ }^{3,4}$ (D) Hans H. C. M. Savelberg ${ }^{5,6}$ (D) - Simone J. P. M. Eussen ${ }^{2,7}$ (D) \\ Pieter C. Dagnelie ${ }^{1,2}$ (1) - Nicolaas C. Schaper ${ }^{1,2,3}$ (D) - Miranda T. Schram ${ }^{1,2,8,9}$ (i) $\cdot$ Carla J. H. van der Kallen ${ }^{1,2}$ (1) \\ Marleen M. J. van Greevenbroek ${ }^{1,2}$ (D) $\cdot$ Anke Wesselius $^{6,10}$ (D) $\cdot$ Casper G. Schalkwijk ${ }^{1,2}$ (D) A Abraham A. Kroon ${ }^{1,2,9}$ (D) \\ Ronald M. A. Henry ${ }^{1,2,9} \cdot$ Coen D. A. Stehouwer ${ }^{1,2}$ (D)
}

Received: 7 October 2021 / Accepted: 28 October 2021 / Published online: 4 February 2022

(C) The Author(s) 2022

\begin{abstract}
Aims/hypothesis Biomarkers of endothelial dysfunction and low-grade inflammation are important in the pathogenesis of CVD and can potentially be modified by physical activity and sedentary behaviour. Effects of physical activity on biomarkers of endothelial dysfunction may be especially prominent in type 2 diabetes.

Methods In the population-based Maastricht Study $(n=2363,51.5 \%$ male, $28.3 \%$ type 2 diabetes, $15.1 \%$ prediabetes [defined as impaired glucose tolerance and impaired fasting glucose]), we determined biomarkers of endothelial dysfunction and lowgrade inflammation, and combined $z$ scores were calculated. Physical activity and sedentary behaviour were measured by activPAL. Linear regression analyses were used with adjustment for demographic, lifestyle and cardiovascular risk factors.

Results The association between total, light, moderate-to-vigorous and vigorous intensity physical activity and sedentary time on the one hand and biomarkers of endothelial dysfunction on the other were generally significant and were consistently stronger in prediabetes and type 2 diabetes as compared with normal glucose metabolism status ( $p$ for interaction $<0.05$ ). Associations between physical activity and sedentary behaviour on the one hand and low-grade inflammation on the other were also significant and were similar in individuals with and without (pre)diabetes ( $p$ for interaction $>0.05$ ).

Conclusions/interpretation Physical activity and sedentary behaviour are associated with biomarkers of endothelial dysfunction and low-grade inflammation. For biomarkers of endothelial dysfunction, associations between physical activity and sedentary behaviour were consistently stronger in (pre)diabetes than in normal glucose metabolism. Whether increasing physical activity or decreasing sedentary time can positively influence biomarkers of endothelial dysfunction in individuals with prediabetes and type 2 diabetes requires further study.
\end{abstract}

Coen D. A. Stehouwer

cda.stehouwer@mumc.nl

1 Department of Internal Medicine, Maastricht University Medical Center+, Maastricht, the Netherlands

2 CARIM School for Cardiovascular Diseases, Maastricht University, Maastricht, the Netherlands

3 CAPHRI Care and Public Health Research Institute, Maastricht University, Maastricht, the Netherlands

4 Department of Social Medicine, Maastricht University, Maastricht, the Netherlands

5 Department of Nutrition and Movement Science, Maastricht University, Maastricht, the Netherlands
$6 \quad$ NUTRIM School for Nutrition and Translational Research in Metabolism, Maastricht University, Maastricht, the Netherlands

7 Department of Epidemiology, Maastricht University, Maastricht, the Netherlands

8 MHeNS School for Mental Health and Neuroscience, Maastricht University, Maastricht, the Netherlands

9 Heart and Vascular Center, Maastricht University Medical Center+, Maastricht, the Netherlands

10 Department of Complex Genetics and Epidemiology, Maastricht University, Maastricht, the Netherlands 


\section{Research in context}

\section{What is already known about this subject?}

- Research has shown that physical activity is inversely associated with biomarkers of endothelial dysfunction and low-grade inflammation

- In individuals with type 2 diabetes, the effects of physical activity on biomarkers of endothelial dysfunction may be especially prominent

\section{What is the key question?}

- What is the relationship between both the amount and pattern of physical activity and sedentary behaviour on the one hand and biomarkers of endothelial dysfunction and low-grade inflammation on the other, especially in individuals with type 2 diabetes?

\section{What are the new findings?}

- There was an inverse association between physical activity and regularity of moderate-to-vigorous intensity physical activity with biomarkers of endothelial dysfunction and low-grade inflammation

- Sedentary time was associated with biomarkers of endothelial dysfunction, whereas sedentary time, prolonged sedentary bouts (both positively) and sedentary breaks (inversely) were all associated with low-grade inflammation

- Associations of physical activity (all intensities) and sedentary time with biomarkers of endothelial dysfunction were consistently stronger in individuals with prediabetes and type 2 diabetes than in individuals with normal glucose metabolism

\section{How might this impact on clinical practice in the foreseeable future?}

- Increasing awareness of the importance of light intensity physical activity and sedentary time may provide an easy and accessible treatment to decrease biomarkers of endothelial dysfunction and low-grade inflammation, especially in individuals with prediabetes and type 2 diabetes

Keywords Cohort study - Diabetes mellitus type 2 - Endothelial dysfunction · Low-grade inflammation · Physical activity · Sedentary behaviour

$\begin{array}{ll}\text { Abbreviations } \\ \text { 2hPG } & \text { 2 h plasma glucose } \\ \text { FPG } & \text { Fasting plasma glucose } \\ \text { hs-CRP } & \text { High-sensitivity C-reactive protein } \\ \text { SAA } & \text { Serum amyloid A } \\ \text { sE-selectin } & \text { Soluble E-selectin } \\ \text { sICAM-1 } & \text { Soluble intercellular adhesion molecule-1 } \\ \text { sVCAM-1 } & \text { Soluble vascular adhesion molecule-1 } \\ \text { vWF } & \text { von Willebrand factor }\end{array}$

\section{Introduction}

Biomarkers of endothelial dysfunction and low-grade inflammation play an important role in the pathogenesis of CVD [1-6]. Lifestyle factors, notably physical activity and diet, are thought to be important modifiable risk factors for the development of endothelial dysfunction and low-grade inflammation [7-9]. Physical activity in particular has been shown to be inversely related to biomarkers of endothelial dysfunction and low-grade inflammation [7, 9-17], possibly because physical activity increases blood flow and shear stress, which improves NO bioavailability [7] and because physical activity reduces visceral adipose tissue, which in turn reduces low-grade inflammation $[8,18]$. The effects of physical activity on biomarkers of endothelial dysfunction may be especially prominent in individuals with type 2 diabetes mellitus. Hyperglycaemia impairs microvascular endothelial function and, specifically, reduces NO availability [19, 20]. Additionally, microvascular endothelial dysfunction (of which these biomarkers are a proxy) is thought to worsen hyperglycaemia through impairment of both insulin-induced glucose uptake and insulin secretion, thus establishing a vicious cycle of biomarkers of endothelial dysfunction and hyperglycaemia [20].

Physical activity is a complex behaviour in which several dimensions can be recognised, such as amount, intensity (light, moderate and vigorous) and weekly pattern. In addition, sedentary behaviour should be taken into account, and may be related to adverse health outcomes independently of 
moderate-to-vigorous intensity physical activity. Such distinctions may be relevant to provide public health guidelines that are more detailed than weekly amount of moderate-tovigorous intensity physical activity [21].

In view of the above, in a large population-based cohort with an oversampling of type 2 diabetes, we investigated the relationship between both the amount and pattern of physical activity and sedentary behaviour on the one hand and biomarkers of endothelial dysfunction and low-grade inflammation on the other. Specifically, we focused on the influence of prediabetes (defined as impaired glucose tolerance and impaired fasting glucose) and type 2 diabetes on these associations.

\section{Methods}

\section{Study population}

We used data from The Maastricht Study, an observational prospective population-based cohort study. The rationale and methodology have been described previously [22].

In brief, the study focuses on the aetiology, pathophysiology, complications and comorbidities of type 2 diabetes and is characterised by an extensive phenotyping approach. Eligible participants were individuals between 40 and 75 years of age and living in the southern part of the Netherlands. Participants were recruited through mass media campaigns and from the municipal registries and the regional Diabetes Patient Registry via mailings. Recruitment was stratified according to known type 2 diabetes status, with an oversampling of individuals with type 2 diabetes, for reasons of efficiency. This study included cross-sectional data from 3451 participants, who completed the baseline survey between November 2010 and September 2013. The examinations of each participant were performed within a time window of 3 months.

The study was approved by the institutional medical ethical committee (NL31329.068.10) and the Minister of Health, Welfare and Sport of the Netherlands (permit no. 131088105234-PG). All participants gave written informed consent.

\section{Measurements}

Markers of low-grade inflammation and biomarkers of endothelial dysfunction Plasma biomarkers of low-grade inflammation (high-sensitivity C-reactive protein [hs-CRP], serum amyloid A [SAA], soluble intercellular adhesion molecule-1 [sICAM-1], IL-6, IL-8, TNF- $\alpha$ ) and biomarkers of endothelial dysfunction (sICAM-1, soluble vascular adhesion molecule-1 [sVCAM-1], soluble E-selectin [sE-selectin]) of the first 866 individuals of The Maastricht Study were measured in EDTA plasma samples with commercially available 4-plex sandwich immunoassay kits (Meso Scale Discovery, Rockville, USA) as described previously [23]. From individual 867 onwards, plasma biomarkers were measured in EDTA plasma samples with renewed commercially available 4-plex sandwich immunoassay kits with different standards and antibodies because of a change in the set-up of the ELISA plates measurements by Meso Scale Discovery. For this technique in this study, the intra- and inter-assay per cent CVs were 5.4 and 5.4 for hsCRP, 8.7 and 10.8 for SAA, 10.3 and 8.4 for SICAM-1, 13.2 and 11.9 for IL-6, 7.6 and 5.5 for IL-8, 4.3 and 6.2 for TNF- $\alpha$, 5.0 and 4.7 for sVCAM-1, and 2.9 and 7.4 for sE-selectin, respectively.

Absolute values of plasma biomarkers differed between individuals measured with the initial and renewed 4-plex sandwich immunoassay kits. To realign absolute values measured with the initial 4-plex sandwich immunoassay to the renewed 4-plex sandwich immunoassay, realignment formulas were calculated with Deming regression analyses [24]. In order to do so, 419 of the initial 866 individuals whose biomarkers were measured with the initial 4-plex sandwich immunoassay were also measured with the renewed 4-plex sandwich immunoassay. The endothelial dysfunction von Willebrand factor (vWF) was quantified in citrate plasma using ELISA (Dako, Glostrup, Denmark). The intra- and inter-assay CVs were $3.0 \%$ and $4.3 \%$, respectively.

\section{Physical activity, activity patterns and sedentary behaviour} Physical activity levels were measured using the activPAL3 physical activity monitor (PAL Technologies, Glasgow, UK). The activPAL3 is a small $(53 \times 35 \times 7 \mathrm{~mm})$, lightweight $(15 \mathrm{~g})$ triaxial accelerometer that records movement in the vertical, anteroposterior and mediolateral axes, and also determines posture (sitting or lying, standing and stepping) based on acceleration information. During the first research visit, the device was attached directly to the skin on the front of the right thigh with transparent $3 \mathrm{M}$ Tegaderm tape, after the device had been waterproofed using a nitrile sleeve. Participants were asked to wear the accelerometer for 8 consecutive days, without removing it at any time. To avoid inaccurately identifying non-wear time, participants were asked not to replace the device once removed. Data were uploaded using the activPAL software and processed using customised software written in MATLAB R2018b (MathWorks, Natick, USA) [25]. Data from the first day were excluded from the analysis because participants performed physical function tests at the research centre after the device was attached. In addition, data from the final wear day providing $\leq 14 \mathrm{~h}$ of out-of-bed data were excluded from the analysis. Participants were included if they provided at least 1 valid day ( $\geq 10 \mathrm{~h}$ of out-of-bed data) and at least 6 valid days for the pattern.

We calculated the amount of time per day spent in light intensity physical activity (defined as standing and $<100$ steps/min), moderate-to-vigorous intensity physical activity (defined as $\geq 100 \mathrm{steps} / \mathrm{min}$ ), and vigorous intensity physical 
activity (defined as $\geq 130 \mathrm{steps} / \mathrm{min}$ ) [26]. Total physical activity per day was defined as mean time spent stepping during out-of-bed time. Weekly activity pattern categories based on moderate-to-vigorous intensity physical activity were defined as: insufficiently active, $<150 \mathrm{~min}$ moderate-to-vigorous intensity physical activity/week; and sufficiently active, $\geq 150$ min moderate-to-vigorous intensity physical activity/ week. The sufficiently active category was further subdivided into 'weekend warrior' and regularly active. In accordance with previous research, weekend warriors were defined as participants who did $\geq 50 \%$ of the weekly moderate-tovigorous intensity physical activity on only 1 or 2 days [27]. Regularly active were participants who did their moderate-tovigorous intensity physical activity in $\geq 3$ days. Thus, we defined three groups: (1) insufficiently active $(0-150 \mathrm{~min}$ moderate-to-vigorous intensity physical activity/week); (2) weekend warrior $(\geq 150$ min moderate-to-vigorous intensity physical activity/week with more than $50 \%$ of the moderateto-vigorous intensity physical activity in 1 or 2 days); and (3) regularly active ( $\geq 150 \mathrm{~min}$ moderate-to-vigorous intensity physical activity/week in $\geq 3$ days). Also, we assessed the variation of moderate-to-vigorous intensity physical activity/week per individual as a continuous variable by calculating the $\mathrm{CV}$ ( $\mathrm{CV}=\mathrm{SD} /$ mean, for each individual).

The total amount of sedentary time was based on the sedentary posture (sitting or lying), and calculated as the mean time spent per day in a sedentary position during out-of-bed time. The method used to determine out-of-bed time has been described elsewhere [25]. In brief, an automated algorithm identified out-of-bed and in-bed times on an individual level on multiple days, i.e. different out-of-bed and in-bed times for each day for each participant. The algorithm is based on the number and duration of sedentary periods to identify in-bed times, and on the number and duration of active periods (standing or stepping) to identify out-of-bed times. The algorithm showed high accuracy in determining out-of-bed time compared with self-report, as the intra-class $r$ was 0.79 $(p<0.001)$ and the mean difference in out-of-bed time between both methods was $0.02 \mathrm{~h}$ (1.2 min), with limits of agreement of -1.1 to $1.2 \mathrm{~h}$.

The number of sedentary breaks during out-of-bed time was determined as each transition from a sitting or lying position to standing or stepping, and the mean number of breaks per day was calculated. Sedentary time accumulated in a consecutive period $\geq 30 \mathrm{~min}$ was defined as a prolonged sedentary bout, and the mean number of prolonged sedentary bouts during out-of-bed time per day was calculated.

Covariates Covariates which were extracted from questionnaires included sex, age, level of education, smoking status, energy intake, Dutch healthy diet index, mobility limitation and history of CVD. Smoking status was categorised into never, former, and current smoker. Level of education was assessed by questionnaire and categorised into low (no education, primary education and lower vocational education); medium (general secondary education, general vocational education, and higher secondary and pre-university education); and high (higher vocational education and university). Energy intake and dietary habits were obtained from a validated food frequency questionnaire [28] and calculated as the mean energy intake (kcal) per day and adherence to Dutch healthy diet index [29]. Mobility limitation was obtained from the 36-Item Short Form Health Survey questionnaire and was defined as having difficulty walking $500 \mathrm{~m}$ or climbing a flight of stairs. Prevalent CVD was defined as a self-reported history of myocardial infarction, ischaemic or haemorrhagic stroke, or percutaneous artery angioplasty of, or vascular surgery on, the coronary, abdominal, peripheral or carotid arteries. The use of lipid-modifying, antihypertensive and glucose-lowering medication was assessed during a medication interview [22]. Office systolic BP, ambulatory $24 \mathrm{~h} \mathrm{BP}$, BMI, waist circumference, $\mathrm{HbA}_{1 \mathrm{c}}$, fasting plasma glucose (FPG), $2 \mathrm{~h}$ plasma glucose (2hPG), triacylglycerol and total cholesterol/HDL-cholesterol ratio were determined as described elsewhere [22]. Glucose metabolism status was assessed by medication use and $2 \mathrm{~h}$ OGTT and classified into normal glucose metabolism, prediabetes (defined as impaired glucose tolerance $[\mathrm{FPG}<7.0 \mathrm{mmol} / \mathrm{l}$ and $2 \mathrm{hPG}$ between $\geq 7.8$ and $<11.1 \mathrm{mmol} / \mathrm{l}]$ and impaired fasting glucose [FPG between 6.1 and $6.9 \mathrm{mmol} / 1$ and $2 \mathrm{hPG}<7.8 \mathrm{mmol} / \mathrm{l}]$ ) and type 2 diabetes according to WHO 2006 criteria [30]. More extensive information of the covariates has been described previously [22].

\section{Statistical analyses}

All data were analysed using IBM SPSS software version 25.0 for Windows (IBM, Armonk, NY). Characteristics of the total study population and according to moderate-to-vigorous intensity physical activity patterns were summarised as mean (SD) or as percentages or as median (IQR) (in case of a skewed distribution). To increase statistical efficiency and reduce multiple testing, an endothelial dysfunction $z$ score and low-grade inflammation $z$ score was calculated.

For endothelial dysfunction we combined: SICAM-1, sVCAM-1, sE-selectin and vWF.

For low-grade inflammation we combined: hs-CRP, IL-6, IL-8, TNF- $\alpha$, SAA and SICAM-1.

First, we calculated the $z$ scores for all individual biomarkers [(individual value minus whole study population mean value)/whole study population $\mathrm{SD}$, thus resulting in a standardised variable ranging from approximately -2.5 to $+2.5 \mathrm{SD}$ with a mean of 0 ].

All the individual $z$ scores from biomarkers share the same unit. They were averaged, resulting in one single endothelial dysfunction or low-grade inflammation score, 
which was subsequently standardised. sICAM-1 was included in both biomarker scores as it is expressed by both monocytes and the endothelium [2, 31-33]. A higher score indicates more biomarkers of endothelial dysfunction or more low-grade inflammation.

Associations between physical activity (total, light intensity, moderate-to-vigorous intensity combined and vigorous intensity separately), sedentary time/breaks, prolonged sedentary bouts and physical activity pattern (independent variables) and biomarkers of endothelial dysfunction and low-grade inflammation scores (dependent variables) were examined with the use of multivariable linear regression models. Models 1 and 2 are about potential confounding, and model 3 is about potential confounding/mediation. Model 1 was adjusted for age, sex and glucose metabolism status; model 2 was additionally adjusted for smoking status, Dutch healthy diet index and level of education; model 3 was additionally adjusted for history of CVD, BMI, office systolic BP, mobility limitations (yes/no), total cholesterol/HDL-cholesterol ratio, triacylglycerol, lipidmodifying medication and antihypertensive medication. The time out-of-bed can be defined as sedentary time, light intensity and moderate-to-vigorous intensity physical activity. Therefore, it may be that sedentary time is a confounder/mediator for moderate-to-vigorous intensity and vigorous intensity physical activity in the above analyses (model 4a). Sedentary behaviour and light intensity physical activity were further adjusted for moderate-to-vigorous intensity physical activity, as a possible confounder/mediator, in the above analyses (model $4 a$ ). Finally, we performed interaction analyses for sex, age and glucose metabolism status in model 3.

Additional analyses Next, we conducted several sensitivity analyses: we excluded individuals with hs-CRP $>10 \mathrm{mg} / \mathrm{l}$; we replaced BMI with waist circumference; we excluded individuals with $<6$ days of activPAL; and we replaced office systolic BP with $24 \mathrm{~h}$ BP. Finally, because biomarkers of endothelial dysfunction and low-grade inflammation are thought to be related processes, they may act as mediators in the above analyses. To test whether this was the case, we adjusted associations with biomarkers of endothelial dysfunction for low-grade inflammation, and vice versa (model $4 \mathrm{~b}$ ). Finally, for the interpretation of the interaction analyses with glucose metabolism status, we performed the interaction analyses described above for glucose metabolism status with $\mathrm{HbA}_{1 \mathrm{c}}$ and FPG and $2 \mathrm{hPG}$ values of the OGTT.

For all analyses, a $p$ value $<0.05$ was considered statistically significant.

\section{Results}

The study population for all analyses, except those for activity patterns and stratified by glucose metabolism status, consisted of 2363 participants. We excluded 810 with no accelerometer measurements, 38 with missing biomarkers of endothelial dysfunction or low-grade inflammation and 240 who had other missing data (confounders) from the overall study population ( $n=3451$ ). Activity pattern analyses were done in 2015 participants, as 348 participants were additionally excluded because of accelerometer measurements of less than 6 days. For the analyses stratified by glucose metabolism status, we excluded other types of diabetes. In total we performed the stratified analysis with 2336 participants. We compared the baseline characteristics of the included and excluded populations and found that the characteristics were similar (electronic supplementary material [ESM] Table 1).

Table 1 shows the characteristics of the total study population and according to the pattern of physical activity. The insufficiently actives, as compared with the more active participants, were older, were more often current smokers, more often had mobility limitations, more often had type 2 diabetes, more often used medication, and had higher levels of biomarkers of endothelial dysfunction and low-grade inflammation.

After adjustment for demographic, lifestyle and cardiovascular risk factors, more physical activity (total and all intensities of physical activity) and more sedentary breaks were inversely associated with biomarkers of endothelial dysfunction (standardised $\beta[95 \% \mathrm{CI}]$, total: $-0.11[-0.15,-0.07]$; light: $-0.05[-0.09,-0.02]$; moderate-to-vigorous: -0.09 $[-0.13,-0.05]$; vigorous: $-0.04[-0.08,-0.004]$; sedentary breaks: $-0.04[-0.08,-0.002]$ ) (Table 2, model 3). Additionally, more sedentary time and more prolonged sedentary bouts were positively associated with biomarkers of endothelial dysfunction (sedentary time: 0.08 [0.04, 0.12]; prolonged sedentary bouts: $0.06[0.02,0.10]$ ) (Table 2 , model 3).

After adjustment for demographic, lifestyle and cardiovascular risk factors, more physical activity (total and all intensities of physical activity) and more sedentary breaks were inversely associated with the low-grade inflammation score (total: $-0.11[-0.15,-0.08]$; light: $-0.07[-0.11,-0.04]$; moderate-to-vigorous: $-0.11[-0.15,-0.07]$; vigorous: -0.07 $[-0.10,-0.03]$; sedentary breaks: $-0.05[-0.09,-0.02])$ (Table 2, model 3). Additionally, more sedentary time and more prolonged sedentary bouts were positively associated with the low-grade inflammation score (sedentary time: 0.08 $[0.05,0.12]$; prolonged sedentary bouts: $0.08[0.04,0.11]$ ) (all $p<0.05$; Table 2, model 3).

To investigate the mutual independence of the above associations, we adjusted light intensity physical activity and sedentary behaviour for moderate-to-vigorous intensity physical activity, and moderate-to-vigorous intensity and vigorous intensity physical activity for sedentary time. For biomarkers of endothelial dysfunction score, only moderate-to-vigorous intensity physical activity and sedentary time remained 
Table 1 Descriptive characteristics of the study population according to physical activity pattern

\begin{tabular}{|c|c|c|c|c|}
\hline \multirow[b]{2}{*}{ Characteristic } & \multirow[b]{2}{*}{$\begin{array}{l}\text { Total population } \\
(n=2363)\end{array}$} & \multicolumn{3}{|c|}{ Physical activity pattern $(n=2015)$} \\
\hline & & $\begin{array}{l}\text { Insufficiently active } \\
(n=173)\end{array}$ & $\begin{array}{l}\text { Weekend warrior } \\
(n=494)\end{array}$ & $\begin{array}{l}\text { Regularly active } \\
(n=1348)\end{array}$ \\
\hline Age (years) & $61.0(55.0-66.0)$ & $64.0(59.0-70.0)$ & $63.0(56.0-67.0)$ & $60.0(54.0-66.0)$ \\
\hline Sex $(\%$ male $)$ & 51.5 & 76.3 & 57.3 & 44.6 \\
\hline \multicolumn{5}{|l|}{ Education level (\%) } \\
\hline Low & 33.5 & 50.9 & 33.0 & 31.1 \\
\hline Medium & 28.1 & 28.9 & 24.3 & 28.6 \\
\hline High & 38.4 & 20.2 & 42.7 & 40.4 \\
\hline \multicolumn{5}{|l|}{ Smoking status $(\%)$} \\
\hline Current & 12.5 & 24.9 & 10.1 & 10.3 \\
\hline Former & 52.2 & 53.2 & 52.4 & 52.0 \\
\hline Never & 35.3 & 22.0 & 37.4 & 37.7 \\
\hline \multicolumn{5}{|l|}{ Alcohol consumption (\%) } \\
\hline None & 17.6 & 28.9 & 14.0 & 16.9 \\
\hline Low & 57.0 & 54.9 & 56.7 & 57.3 \\
\hline High & 25.4 & 16.2 & 29.4 & 25.8 \\
\hline \multicolumn{5}{|l|}{ Mobility limitations (\%) } \\
\hline Yes & 20.3 & 46.8 & 17.8 & 17.4 \\
\hline No & 79.7 & 53.2 & 82.2 & 82.6 \\
\hline BMI $\left(\mathrm{kg} / \mathrm{m}^{2}\right)$ & $27.02(4.54)$ & $30.11(5.51)$ & $26.82(4.09)$ & $26.42(4.11)$ \\
\hline History of CVD (\%) & 16.6 & 30.1 & 16.4 & 15.0 \\
\hline \multicolumn{5}{|l|}{ Diabetes status (\%) } \\
\hline Normal & 55.5 & 23.1 & 55.7 & 61.6 \\
\hline Impaired & 15.1 & 10.4 & 16.8 & 14.8 \\
\hline Type 2 diabetes & 28.3 & 64.7 & 26.7 & 22.4 \\
\hline Other type of diabetes & 1.1 & 1.7 & 0.8 & 1.2 \\
\hline Antihypertensive medication use (\%) & 41.4 & 69.4 & 42.7 & 37.8 \\
\hline Lipid-modifying medication use (\%) & 37.7 & 64.2 & 37.9 & 32.9 \\
\hline Glucose-lowering medication use (\%) & 23.7 & 58.4 & 21.7 & 18.4 \\
\hline Total cholesterol/HDL-cholesterol ratio & $3.38(2.76-4.19)$ & $3.64(3.00-4.60)$ & $3.45(2.82-4.29)$ & $3.28(2.70-4.06)$ \\
\hline Triacylglycerol (mmol/l) & $1.22(0.89-1.72)$ & $1.54(1.12-2.14)$ & $1.25(0.90-1.75)$ & $1.16(0.86-1.61)$ \\
\hline Dutch healthy diet index & $83.73(14.67)$ & $78.19(14.80)$ & $84.39(13.63)$ & $85.08(14.81)$ \\
\hline Valid days $(n)$ & $7.0(6.00-7.00)$ & $7.00(6.00-7.00)$ & $7.0(6.00-7.00)$ & $7.0(6.00-7.00)$ \\
\hline Sedentary time (h/day) & $9.42(1.67)$ & $10.96(1.51)$ & $9.66(1.44)$ & $9.09(1.55)$ \\
\hline Total physical activity (h/day) & $1.96(1.49-2.44)$ & $1.01(0.81-1.21)$ & $1.85(1.50-2.25)$ & $2.09(1.68-2.55)$ \\
\hline Light intensity physical activity (h/day) & $5.41(1.52)$ & $4.12(1.39)$ & $5.07(1.30)$ & $5.69(1.43)$ \\
\hline $\begin{array}{l}\text { Moderate-to-vigorous intensity physical activity } \\
\text { (h/day) }\end{array}$ & $0.85(0.58-1.15)$ & $0.27(0.19-0.31)$ & $0.84(0.64-1.10)$ & $0.94(0.68-1.22)$ \\
\hline Vigorous intensity physical activity (h/day) & $0.09(0.04-0.19)$ & $0.02(0.01-0.03)$ & $0.09(0.05-0.18)$ & $0.10(0.06-0.21)$ \\
\hline Sedentary breaks $(n /$ day $)$ & $37.45(8.58)$ & $33.17(8.21)$ & $35.50(7.63)$ & $38.51(8.25)$ \\
\hline Prolonged sedentary bouts ( $n /$ day) & $4.86(3.71-5.86)$ & $6.29(5.17-7.17)$ & $5.29(4.33-6.14)$ & $4.46(3.50-5.50)$ \\
\hline sICAM-1 (ng/ml) & $355.62(98.26)$ & $402.95(144.46)$ & $353.75(94.21)$ & $348.68(85.17)$ \\
\hline sVCAM-1 (ng/ml) & $428.50(100.61)$ & 471.35 (135.04) & $432.68(99.96)$ & $421.59(91.75)$ \\
\hline sE-selectin (ng/ml) & $117.81(66.46)$ & $154.91(112.77)$ & $114.12(58.05)$ & $112.39(59.95)$ \\
\hline von Willebrand factor (\%) & $132.20(47.84)$ & $154.03(52.48)$ & $134.60(51.34)$ & $128.32(44.64)$ \\
\hline Plasma hs-CRP (mg/l) & $1.22(0.61-2.72)$ & $1.99(1.01-4.04)$ & $1.21(0.64-2.84)$ & $1.12(0.57-2.45)$ \\
\hline Plasma SAA $(\mu \mathrm{g} / \mathrm{ml})$ & $3.36(2.09-5.45)$ & $3.48(2.10-6.89)$ & $3.30(2.14-5.31)$ & $3.36(2.04-5.36)$ \\
\hline Plasma IL-6 (pg/ml) & $0.58(0.39-0.90)$ & $0.89(0.65-1.42)$ & $0.57(0.42-0.87)$ & $0.55(0.37-0.84)$ \\
\hline Plasma IL-8 (pg/ml) & $4.11(3.25-5.29)$ & $4.83(3.89-6.42)$ & $4.23(3.34-5.27)$ & $3.97(3.19-5.13)$ \\
\hline Plasma TNF- $\alpha(\mathrm{pg} / \mathrm{ml})$ & $2.20(1.89-2.56)$ & $2.50(2.19-3.00)$ & $2.18(1.86-2.55)$ & $2.16(1.87-2.51)$ \\
\hline
\end{tabular}

Values are means $(\mathrm{SD})$ or median $(\mathrm{Q} 1-\mathrm{Q} 3)$, unless stated otherwise

associated (Table 2, model 4a). For the low-grade inflammation score, associations remained similar for light intensity physical activity, moderate-to-vigorous intensity and vigorous intensity physical activity, sedentary time, sedentary breaks and prolonged sedentary bouts (Table 2 , model $4 a$ ).

After adjustment for demographic, lifestyle and cardiovascular risk factors, regularly actives and weekend warriors, as compared with the insufficiently actives (as reference), had comparably lower biomarkers of endothelial dysfunction and low-grade inflammation scores (biomarkers of endothelial dysfunction: weekend warriors $-0.28[-0.44,-0.11]$; regularly actives $-0.32[-0.47,-0.16]$, low-grade inflammation: weekend warriors $-0.22[-0.38,-0.07]$; regularly actives $-0.26[-0.41$, -0.11]) (Table 3, model 3). However, the CV of the weekly 


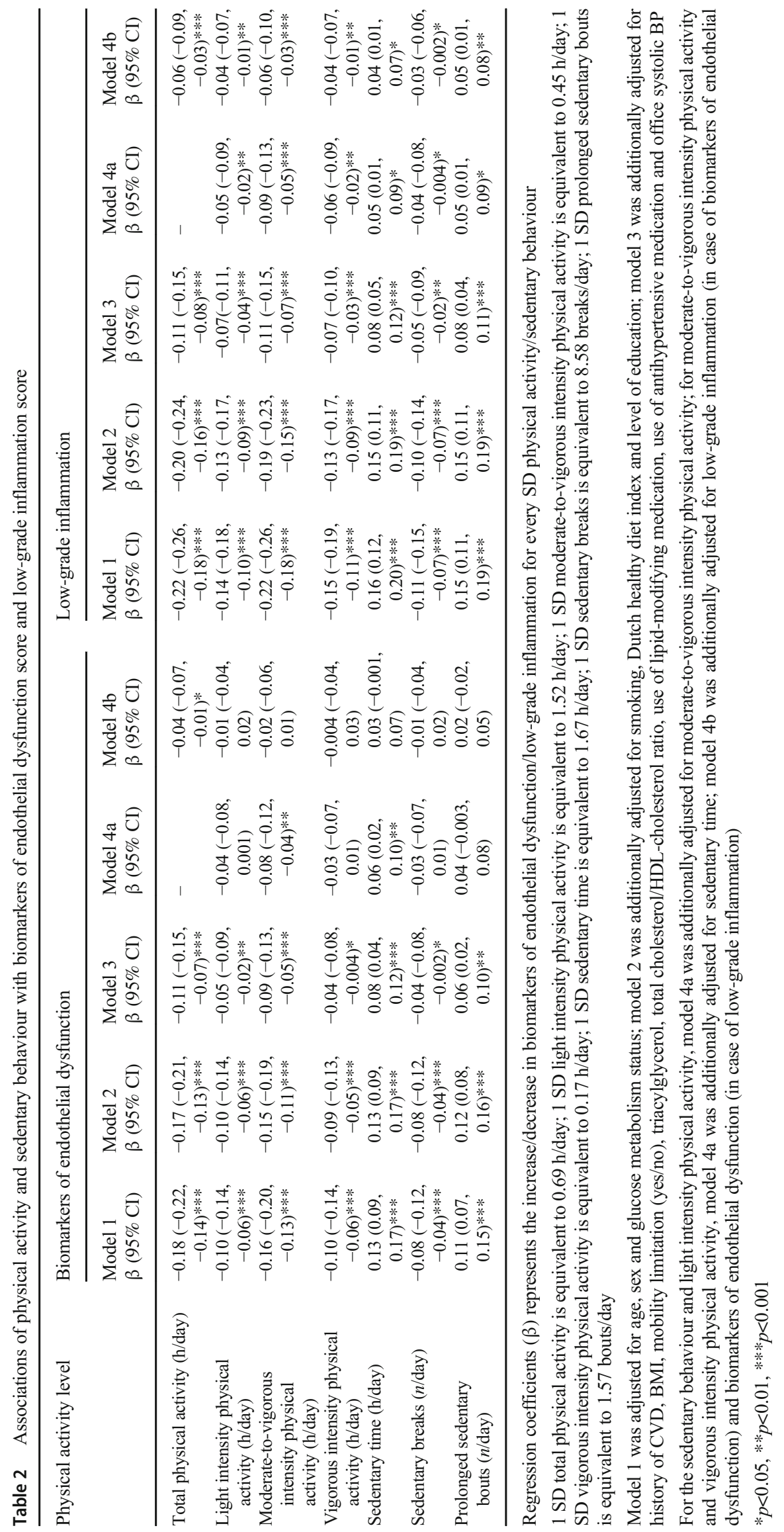




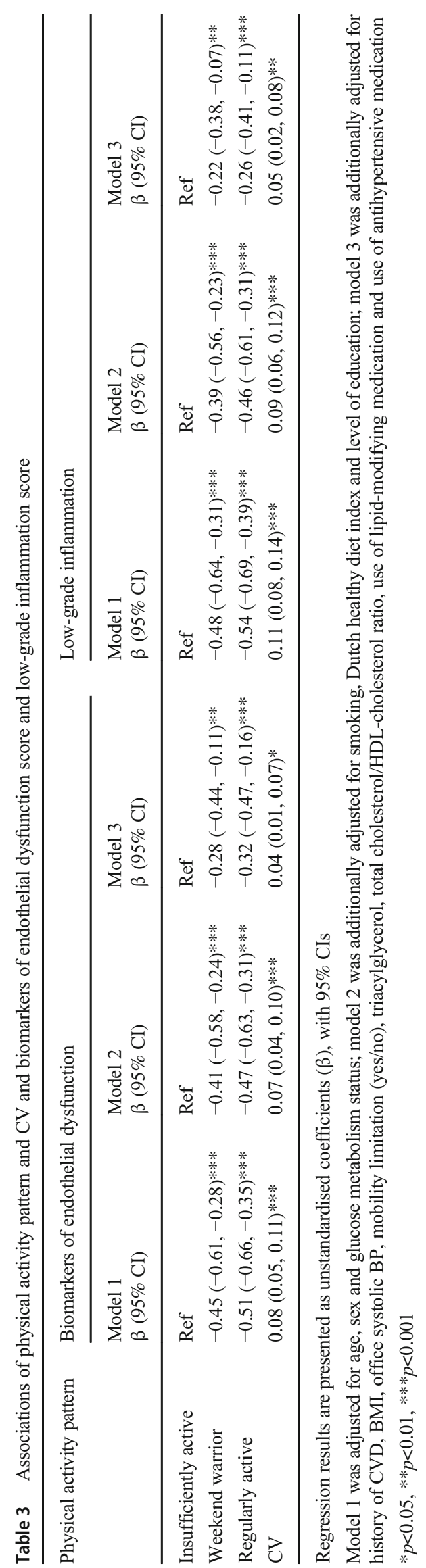


Table 4 Associations of physical activity and sedentary time with biomarkers of endothelial dysfunction, stratified by glucose metabolism status

\begin{tabular}{|c|c|c|c|}
\hline \multirow[t]{2}{*}{ Physical activity level } & \multicolumn{3}{|c|}{ Biomarkers of endothelial dysfunction } \\
\hline & $\begin{array}{l}\text { Model } 1 \\
\beta(95 \% \mathrm{CI})\end{array}$ & $\begin{array}{l}\text { Model } 2 \\
\beta(95 \% \text { CI })\end{array}$ & $\begin{array}{l}\text { Model } 3 \\
\beta(95 \% \mathrm{CI})\end{array}$ \\
\hline \multicolumn{4}{|l|}{ Total physical activity } \\
\hline Normal glucose metabolism & $-0.12(-0.17,-0.06)^{* * *}$ & $-0.11(-0.17,-0.06)^{* * *}$ & $-0.06(-0.12,-0.01)^{*}$ \\
\hline Prediabetes & $-0.20(-0.30,-0.10)^{* * *}$ & $-0.21(-0.31,-0.10)^{* * *}$ & $-0.16(-0.26,-0.05)^{* *}$ \\
\hline $\mathrm{T} 2 \mathrm{D}$ & $-0.27(-0.34,-0.19)^{* * *}$ & $-0.25(-0.32,-0.17)^{* * *}$ & $-0.14(-0.22,-0.06)^{* * *}$ \\
\hline \multicolumn{4}{|l|}{ Light intensity physical activity } \\
\hline Normal glucose metabolism & $-0.02(-0.08,0.03)$ & $-0.02(-0.08,0.03)$ & $0.02(-0.04,0.07)$ \\
\hline Prediabetes & $-0.15(-0.26,-0.04)^{* *}$ & $-0.16(-0.27,-0.05)^{* *}$ & $-0.17(-0.27,-0.06)^{* *}$ \\
\hline $\mathrm{T} 2 \mathrm{D}$ & $-0.17(-0.25,-0.10)^{* * *}$ & $-0.17(-0.25,-0.10)^{* * *}$ & $-0.10(-0.18,-0.03)^{* *}$ \\
\hline \multicolumn{4}{|c|}{ Moderate-to-vigorous intensity physical activity } \\
\hline Normal glucose metabolism & $-0.13(-0.18,-0.07)^{* * *}$ & $-0.12(-0.17,-0.07)^{* * *}$ & $-0.08(-0.13,-0.02)^{* *}$ \\
\hline Prediabetes & $-0.17(-0.27,-0.07)^{* *}$ & $-0.16(-0.26,-0.06)^{* *}$ & $-0.11(-0.21,-0.001)^{*}$ \\
\hline $\mathrm{T} 2 \mathrm{D}$ & $-0.24(-0.31,-0.16)^{* * *}$ & $-0.21(-0.28,-0.13)^{* * *}$ & $-0.10(-0.18,-0.02)^{*}$ \\
\hline \multicolumn{4}{|c|}{ Vigorous intensity physical activity } \\
\hline Normal glucose metabolism & $-0.11(-0.16,-0.05)^{* * *}$ & $-0.10(-0.16,-0.05)^{* * *}$ & $-0.06(-0.11,-0.002)^{*}$ \\
\hline Prediabetes & $-0.10(-0.20,0.01)$ & $-0.09(-0.19,0.02)$ & $-0.03(-0.13,0.08)$ \\
\hline $\mathrm{T} 2 \mathrm{D}$ & $-0.15(-0.22,-0.07)^{* * *}$ & $-0.12(-0.20,-0.05)^{* *}$ & $-0.05(-0.13,0.02)$ \\
\hline \multicolumn{4}{|l|}{ Sedentary time } \\
\hline Normal glucose metabolism & $0.06(0.001,0.10)^{*}$ & $0.06(0.003,0.12)^{*}$ & $0.02(-0.04,0.07)$ \\
\hline Prediabetes & $0.20(0.10,0.31)^{* * *}$ & $0.22(0.11,0.33)^{* * *}$ & $0.21(0.10,0.31)^{* * *}$ \\
\hline $\mathrm{T} 2 \mathrm{D}$ & $0.18(0.11,0.26)^{* * *}$ & $0.18(0.10,0.25)^{* * *}$ & $0.10(0.02,0.17)^{*}$ \\
\hline
\end{tabular}

Regression coefficients $(\beta)$ represents the increase/decrease in biomarkers of endothelial dysfunction for every SD physical activity/sedentary behaviour $1 \mathrm{SD}$ total physical activity is equivalent to $0.67 \mathrm{~h} /$ day, $1 \mathrm{SD}$ light intensity physical activity is equivalent to $1.48 \mathrm{~h} /$ day, $1 \mathrm{SD}$ moderate-to-vigorous intensity physical activity is equivalent to $0.43 \mathrm{~h} /$ day, $1 \mathrm{SD}$ vigorous intensity physical activity is equivalent to $0.16 \mathrm{~h} /$ day, $1 \mathrm{SD}$ sedentary time is equivalent to $1.60 \mathrm{~h} /$ day

Model 1 was adjusted for age and sex; model 2 was additionally adjusted for smoking, Dutch healthy diet index and level of education; model 3 was additionally adjusted for history of CVD, BMI, office systolic BP, mobility limitation (yes/no), triacylglycerol, total cholesterol/HDL-cholesterol ratio, use of lipid-modifying medication and use of antihypertensive medication

${ }^{*} p<0.05, * * p<0.01, * * * p<0.001$

T2D, type 2 diabetes

amount of moderate-to-vigorous intensity physical activity (regularity) was positively associated with biomarkers of endothelial dysfunction and low-grade inflammation scores (biomarkers of endothelial dysfunction: 0.04 [0.01, 0.07], low-grade inflammation: 0.05 [0.02, 0.08]) (Table 3, model 3).

Associations of physical activity and sedentary behaviour with individual biomarkers of endothelial dysfunction and low-grade inflammation are shown in ESM Tables 210. In general, associations were similar to those with biomarkers of endothelial dysfunction and low-grade inflammation scores.

Associations between light intensity physical activity and prolonged sedentary bouts on the one hand and low-grade inflammation (but not biomarkers of endothelial dysfunction) on the other were stronger in women than in men ( $p$ for interaction $<0.05$; ESM Table 11 shows stratified analyses). There was no interaction with age.
Associations between total physical activity, light intensity physical activity, moderate-to-vigorous intensity physical activity, vigorous intensity physical activity and sedentary time on the one hand and biomarkers of endothelial dysfunction (but not low-grade inflammation) on the other were in general consistently stronger in individuals with prediabetes and type 2 diabetes than in those without ( $p$ for interaction $<0.05$; standardised $\beta[95 \% \mathrm{CI}]$ total physical activity: $-0.16[-0.26$, $-0.05],-0.14[-0.22,-0.06],-0.06[-0.12,-0.01]$; light intensity physical activity: $-0.17[-0.27,-0.06],-0.10[-0.18$, $-0.03], 0.02$ [-0.04, 0.07]; moderate-to-vigorous intensity physical activity: $-0.11[-0.21,-0.001],-0.10[-0.18$, $-0.02],-0.08[-0.13,-0.02]$; vigorous intensity physical activity: $-0.03[-0.13,0.08],-0.05[-0.13,0.02],-0.06[-0.11$, $-0.002]$; sedentary time: 0.21 [0.10, 0.31$], 0.10$ [0.02, 0.17], $0.02[-0.04,0.07])$; Table 4 shows these stratified analyses. When we replaced glucose metabolism status with $\mathrm{HbA}_{1 \mathrm{c}}$, 
FPG or $2 \mathrm{hPG}$ in the interaction analyses with biomarkers of endothelial dysfunction, we observed significant interactions for total physical activity (FPG and 2hPG), light intensity physical activity $\left(\mathrm{HbA}_{1 \mathrm{c}}, \mathrm{FPG}\right.$ and $\left.2 \mathrm{hPG}\right)$, moderate-to-vigorous intensity physical activity (FPG and 2hPG) and sedentary time (FPG and $2 \mathrm{hPG}$ ), but not for vigorous intensity physical activity (ESM Tables 12-14).

\section{Additional analyses}

To test the robustness of the above results, we did several sensitivity analyses. In general, results remained similar when we excluded participants with hs-CRP> $10 \mathrm{mg} / \mathrm{l}$ (ESM Tables 15 and 16); when we replaced BMI with waist circumference (ESM Tables 17 and 18); when we replaced office systolic BP with 24 h systolic BP (ESM Tables 19 and 20); and when we excluded participants with less than 6 days of activPAL (2015 individuals) (ESM Table 21).

Finally, from a pathophysiological point of view, biomarkers of endothelial dysfunction and low-grade inflammation are strongly interlinked [34, 35]. Indeed, when we adjusted associations with biomarkers of endothelial dysfunction as outcome for the low-grade inflammation score, or vice versa, associations of physical activity intensity or sedentary behaviour with these outcomes were consistently attenuated (Table 2, model 4b).

\section{Discussion}

This cross-sectional population-based study on the associations between accelerometer-measured physical activity and sedentary behaviour on the one hand and biomarkers of endothelial dysfunction and low-grade inflammation on the other had three main findings. First, all intensities of physical activity and, in addition, regularity of moderate-to-vigorous intensity physical activity were inversely associated with biomarkers of endothelial dysfunction and low-grade inflammation. Second, and independently of moderate-to-vigorous intensity physical activity, sedentary time was associated with biomarkers of endothelial dysfunction, whereas sedentary time, prolonged sedentary bouts (both positively) and sedentary breaks (inversely) were all associated with low-grade inflammation. Third, associations between physical activity (all intensities) and sedentary time on the one hand and biomarkers of endothelial dysfunction on the other were consistently stronger in individuals with prediabetes and type 2 diabetes than in individuals with normal glucose metabolism.

All intensities of physical activity were inversely associated with biomarkers of endothelial dysfunction and low-grade inflammation. In general, our results are consistent with previous studies [12-15, 36-39]. As an important extension to previous publications, the present study had the advantage of a more extensive characterisation of both physical activity on the one hand and biomarkers of endothelial dysfunction and low-grade inflammation on the other. Importantly, we found that regularity (i.e. a lower $\mathrm{CV}$, as a continuous variable) of moderate-to-vigorous intensity activity was associated with less biomarkers of endothelial dysfunction and lowgrade inflammation. We attribute the observation that similar differences were not observed when regularly actives were compared with weekend warriors to the decrease in power associated with analyses with categorical variables.

Independently of moderate-to-vigorous intensity physical activity, sedentary time was associated with biomarkers of endothelial dysfunction, whereas sedentary time, prolonged sedentary bouts (both positively) and sedentary breaks (inversely) were all associated with low-grade inflammation. These results are generally consistent with those of previous studies [11, 40-43].

Physical activity is thought to ameliorate endothelial function through inducing shear stress, which in turn increases activity of endothelial oxide nitric synthase and enhances NO bioavailability [7]. Conversely, sedentary behaviour reduces shear stress, which decreases endothelial oxide nitric synthase and decreases bioavailability of NO [11]. However, the mechanisms linking physical activity and sedentary behaviour to low-grade inflammation are not completely understood. Possible explanations include a reduction in visceral adipose tissue, a reduction of toll-like receptors on immune cells, and changes in the number of (immune) cells $[8,18]$.

We found that associations between physical activity and biomarkers of endothelial dysfunction were consistently stronger in individuals with prediabetes and type 2 diabetes than in individuals with normal glucose metabolism. When analyses were repeated with measures of glucose $\left(\mathrm{HbA}_{1 \mathrm{c}}\right.$, FPG or $2 \mathrm{hPG}$ ), results were mostly but not entirely consistent, which may be due to the play of chance or reflect true differences between (pre)diabetes as a state and glucose measures that are not explained by the adjustments we made, and thus were not identified in this study.

Hyperglycaemia and impairment of microvascular endothelial function are thought to constitute a vicious cycle [20]. We speculate that physical activity, by improving microvascular endothelial function, interrupts this vicious cycle. In short-term studies, the effect on biomarkers of endothelial dysfunction of increasing physical activity has not been found to be larger in individuals with, than in those without, type 2 diabetes [39], but it must be recognised that activity measurements such as those obtained in the current study probably reflect long-term lifestyle habits. Thus, long-term exposures to greater physical activity may be needed to improve endothelial function in individuals with type 2 diabetes. This hypothesis requires further study in long-term intervention trials.

This study had several strengths: we studied a large population-based sample; we studied amount, intensity and 
pattern of physical activity as measured by accelerometry; we used multiple biomarkers of endothelial dysfunction and lowgrade inflammation; and we took extensive measurements of possible confounders, which makes residual confounding less likely. The study also had several limitations. Because of the cross-sectional design, causal inference should be made with great caution, although our results are physiologically plausible. Earlier research showed that poorer health is associated with less physical activity and more sedentary behaviour [44-46]. Next, the use of composite biomarker scores assumes that all biomarkers are equally important in pathophysiological terms. Whether this assumption holds is not completely clear, but all biomarkers used have been shown to be prognostically linked to CVD [3-5], and associations were homogeneous across biomarkers. Furthermore, we have only used the activPAL, which unfortunately does not allow us to distinguish the specific type of activities (e.g. endurance exercise vs strength exercise and subtypes of exercise, such as walking, running, cycling, swimming). All activities are captured as activity by the activPAL, and we were further able to classify activities into light, moderate and vigorous intensity physical activity. Finally, the population consisted only of White participants, which limits generalisability of our findings.

In conclusion, our study shows that, independently of demographic, lifestyle and cardiovascular risk factors, different intensities of physical activity are inversely associated with biomarkers of endothelial dysfunction and low-grade inflammation. Additionally, greater regularity of moderateto-vigorous intensity physical activity is advantageous with regard to biomarkers of endothelial dysfunction and lowgrade inflammation. Sedentary time was directly associated with biomarkers of endothelial dysfunction, and sedentary time, prolonged sedentary bouts (positively) and sedentary breaks (inversely) were associated with low-grade inflammation, and this was independent of moderate-to-vigorous intensity physical activity. Importantly, associations of physical activity and biomarkers of endothelial dysfunction were consistently stronger in prediabetes and type 2 diabetes. Taken together, these results support increasing physical activity and limiting sedentary behaviour as a means to prevent or ameliorate biomarkers of endothelial dysfunction and low-grade inflammation, especially in individuals with prediabetes and type 2 diabetes.

Supplementary Information The online version of this article (https://doi. org/10.1007/s00125-022-05651-3) contains peer-reviewed but unedited supplementary material.

Acknowledgements Some of the data were presented as an abstract at the Annual Dutch Diabetes Research Meeting in 2020.

Data availability The datasets generated during and/or analysed during the current study are not publicly available but are available from the corresponding author on reasonable request.
Funding This study was supported by the European Regional Development Fund via OP-Zuid, the Province of Limburg, the Dutch Ministry of Economic Affairs (grant 310.041), Stichting De Weijerhorst (Maastricht, the Netherlands), the Pearl String Initiative Diabetes (Amsterdam, the Netherlands), the Cardiovascular Center (CVC, Maastricht, the Netherlands), CARIM School for Cardiovascular Diseases (Maastricht, the Netherlands), CAPHRI Care and Public Health Research Institute (Maastricht, the Netherlands), NUTRIM School for Nutrition and Translational Research in Metabolism (Maastricht, the Netherlands), Stichting Annadal (Maastricht, the Netherlands), Health Foundation Limburg (Maastricht, the Netherlands), and by unrestricted grants from Janssen-Cilag BV (Tilburg, the Netherlands), Novo Nordisk Farma BV (Alphen aan den Rijn, the Netherlands), and Sanofi-Aventis Netherlands BV (Gouda, the Netherlands). This project was partly funded through an EFSD award supported by AstraZeneca.

Authors' relationships and activities The authors declare that there are no relationships or activities that might bias, or be perceived to bias, their work.

Contribution statement EJV and CDAS contributed to conception and design, participated in acquisition of data, analysed and interpreted data, drafted the manuscript, revised the manuscript critically for important intellectual content, and provided final approval of the version to be published. AK and RMAH contributed to conception and design, contributed to analyses and interpretation of data, revised the manuscript critically for important intellectual content, and provided final approval of the version to be published. HHCMS, SJPME, PCD, NCS, MTS, CJHvdK, MMJvG, AW, CGS and AAK were involved in the acquisition of data, revised the manuscript critically for important intellectual content and provided final approval of the version to be published. EJV and CDAS are the guarantors of this work and, as such, had full access to all the data in the study and take responsibility for the integrity of the data and the accuracy of the data analysis.

Open Access This article is licensed under a Creative Commons Attribution 4.0 International License, which permits use, sharing, adaptation, distribution and reproduction in any medium or format, as long as you give appropriate credit to the original author(s) and the source, provide a link to the Creative Commons licence, and indicate if changes were made. The images or other third party material in this article are included in the article's Creative Commons licence, unless indicated otherwise in a credit line to the material. If material is not included in the article's Creative Commons licence and your intended use is not permitted by statutory regulation or exceeds the permitted use, you will need to obtain permission directly from the copyright holder. To view a copy of this licence, visit http://creativecommons.org/licenses/by/4.0/.

\section{References}

1. de Jager J, Dekker JM, Kooy A et al (2006) Endothelial dysfunction and low-grade inflammation explain much of the excess cardiovascular mortality in individuals with type 2 diabetes: the Hoorn Study. Arterioscler Thromb Vasc Biol 26(5):1086-1093. https://doi.org/ 10.1161/01.ATV.0000215951.36219.a4

2. Jager A, van Hinsbergh VW, Kostense PJ et al (1999) von Willebrand factor, C-reactive protein, and 5-year mortality in diabetic and nondiabetic subjects: the Hoorn Study. Arterioscler Thromb Vasc Biol 19(12):3071-3078. https://doi.org/10.1161/01. atv.19.12.3071

3. Blankenberg S, Rupprecht HJ, Bickel C et al (2001) Circulating cell adhesion molecules and death in patients with coronary artery 
disease. Circulation 104(12):1336-1342. https://doi.org/10.1161/ hc3701.095949

4. Ridker PM, Hennekens CH, Buring JE, Rifai N (2000) C-reactive protein and other markers of inflammation in the prediction of cardiovascular disease in women. N Engl J Med 342(12):836843. https://doi.org/10.1056/nejm200003233421202

5. Hansson GK (2005) Inflammation, atherosclerosis, and coronary artery disease. N Engl J Med 352(16):1685-1695. https://doi.org/ 10.1056/NEJMra043430

6. Geovanini GR, Libby P (2018) Atherosclerosis and inflammation: overview and updates. Clin Sci (Lond) 132(12):1243-1252. https:// doi.org/10.1042/cs20180306

7. Ribeiro F, Alves AJ, Duarte JA, Oliveira J (2010) Is exercise training an effective therapy targeting endothelial dysfunction and vascular wall inflammation? Int J Cardiol 141(3):214-221. https:// doi.org/10.1016/j.ijcard.2009.09.548

8. Gleeson M, Bishop NC, Stensel DJ, Lindley MR, Mastana SS, Nimmo MA (2011) The anti-inflammatory effects of exercise: mechanisms and implications for the prevention and treatment of disease. Nat Rev Immunol 11(9):607-615. https://doi.org/10.1038/ nri3041

9. Pedersen BK (2006) The anti-inflammatory effect of exercise: its role in diabetes and cardiovascular disease control. Essays Biochem 42:105-117. https://doi.org/10.1042/bse0420105

10. Pinto A, Di Raimondo D, Tuttolomondo A, Buttà C, Milio G, Licata G (2012) Effects of physical exercise on inflammatory markers of atherosclerosis. Curr Pharm Des 18(28):4326-4349. https://doi.org/10.2174/138161212802481192

11. Thosar SS, Johnson BD, Johnston JD, Wallace JP (2012) Sitting and endothelial dysfunction: the role of shear stress. Med Sci Monit 18(12):Ra173-Ra180. https://doi.org/10.12659/msm.883589

12. Lin X, Zhang X, Guo J et al (2015) Effects of Exercise Training on Cardiorespiratory Fitness and Biomarkers of Cardiometabolic Health: A Systematic Review and Meta-Analysis of Randomized Controlled Trials. J Am Heart Assoc 4(7):e002014. https://doi.org/ 10.1161/jaha.115.002014

13. Palmefors H, DuttaRoy S, Rundqvist B, Börjesson M (2014) The effect of physical activity or exercise on key biomarkers in atherosclerosis-a systematic review. Atherosclerosis 235(1):150 161. https://doi.org/10.1016/j.atherosclerosis.2014.04.026

14. Gram AS, Bladbjerg EM, Quist JS, Petersen MB, Rosenkilde M, Stallknecht B (2017) Anti-inflammatory effects of active commuting and leisure time exercise in overweight and obese women and men: A randomized controlled trial. Atherosclerosis 265:318-324. https://doi.org/10.1016/j.atherosclerosis.2017.06.923

15. Abramson JL, Vaccarino V (2002) Relationship between physical activity and inflammation among apparently healthy middle-aged and older US adults. Arch Intern Med 162(11):1286-1292. https:// doi.org/10.1001/archinte.162.11.1286

16. Stolberg CR, Mundbjerg LH, Funch-Jensen P, Gram B, Bladbjerg EM, Juhl CB (2018) Effects of gastric bypass surgery followed by supervised physical training on inflammation and endothelial function: A randomized controlled trial. Atherosclerosis 273:37-44. https://doi.org/10.1016/j.atherosclerosis.2018.04.002

17. Duvivier B, Bolijn JE, Koster A, Schalkwijk CG, Savelberg H, Schaper NC (2018) Reducing sitting time versus adding exercise: differential effects on biomarkers of endothelial dysfunction and metabolic risk. Sci Rep 8(1):8657. https://doi.org/10.1038/ s41598-018-26616-w

18. Hoekstra SP, Bishop NC, Leicht CA (2020) Elevating body temperature to reduce low-grade inflammation: a welcome strategy for those unable to exercise? Exerc Immunol Rev 26:42-55

19. Kaur R, Kaur M, Singh J (2018) Endothelial dysfunction and platelet hyperactivity in type 2 diabetes mellitus: molecular insights and therapeutic strategies. Cardiovasc Diabetol 17(1):121. https://doi. org/10.1186/s12933-018-0763-3
20. Stehouwer CDA (2018) Microvascular Dysfunction and Hyperglycemia: A Vicious Cycle With Widespread Consequences. Diabetes 67(9):1729-1741. https://doi.org/10. 2337/dbi17-0044

21. Bull FC, Al-Ansari SS, Biddle S et al (2020) World Health Organization 2020 guidelines on physical activity and sedentary behaviour. Br J Sports Med 54(24):1451-1462. https://doi.org/10. 1136/bjsports-2020-102955

22. Schram MT, Sep SJ, van der Kallen CJ et al (2014) The Maastricht study: an extensive phenotyping study on determinants of type 2 diabetes, its complications and its comorbidities. Eur J Epidemiol 29(6):439-451. https://doi.org/10.1007/s10654-014-9889-0

23. van Dooren FE, Schram MT, Schalkwijk CG et al (2016) Associations of low grade inflammation and endothelial dysfunction with depression - The Maastricht Study. Brain Behav Immun 56:390-396. https://doi.org/10.1016/j.bbi.2016.03.004

24. van Bussel BCT, Ferreira I, van de Waarenburg MPH et al (2013) Multiple inflammatory biomarker detection in a prospective cohort study: a cross-validation between well-established single-biomarker techniques and an electrochemiluminescense-based multi-array platform. PLoS One 8(3):e58576-e58576. https://doi.org/10.1371/ journal.pone.0058576

25. van der Berg JD, Willems PJ, van der Velde JH et al (2016) Identifying waking time in 24-h accelerometry data in adults using an automated algorithm. J Sports Sci 34(19):1867-1873. https:// doi.org/10.1080/02640414.2016.1140908

26. Tudor-Locke C, Aguiar EJ, Han H et al (2019) Walking cadence (steps/min) and intensity in 21-40 year olds: CADENCE-adults. Int J Behav Nutr Phys Act 16(1):8. https://doi.org/10.1186/s12966019-0769-6

27. Shiroma EJ, Lee IM, Schepps MA, Kamada M, Harris TB (2019) Physical Activity Patterns and Mortality: The Weekend Warrior and Activity Bouts. Med Sci Sports Exerc 51(1):35-40. https:// doi.org/10.1249/mss.0000000000001762

28. van Dongen MC, Wijckmans-Duysens NEG, den Biggelaar LJ et al (2019) The Maastricht FFQ: Development and validation of a comprehensive food frequency questionnaire for the Maastricht study. Nutrition 62:39-46. https://doi.org/10.1016/j.nut.2018.10. 015

29. Looman M, Feskens EJ, de Rijk M et al (2017) Development and evaluation of the Dutch Healthy Diet index 2015. Public Health Nutr 20(13):2289-2299. https://doi.org/10.1017/s136898001700091x

30. World Health Organization (2006) Definition and Diagnosis of Diabetes Mellitus and Intermediate Hyperglycemia: Report of a WHO/IDF Consultation. World Health Organization, Geneva

31. Yudkin JS, Stehouwer CD, Emeis JJ, Coppack SW (1999) Creactive protein in healthy subjects: associations with obesity, insulin resistance, and endothelial dysfunction: a potential role for cytokines originating from adipose tissue? Arterioscler Thromb Vasc Biol 19(4):972-978. https://doi.org/10.1161/01.atv.19.4.972

32. van Bussel BC, Henry RM, Schalkwijk CG et al (2011) Fish consumption in healthy adults is associated with decreased circulating biomarkers of endothelial dysfunction and inflammation during a 6-year follow-up. J Nutr 141(9):1719-1725. https://doi. org/10.3945/jn.111.139733

33. van Bussel BC, Schouten F, Henry RM et al (2011) Endothelial dysfunction and low-grade inflammation are associated with greater arterial stiffness over a 6-year period. Hypertension 58(4):588-595. https://doi.org/10.1161/hypertensionaha.111.174557

34. Borissoff JI, Spronk HM, ten Cate H (2011) The hemostatic system as a modulator of atherosclerosis. N Engl J Med 364(18):17461760. https://doi.org/10.1056/NEJMra1011670

35. Stehouwer CD, Henry RM, Ferreira I (2008) Arterial stiffness in diabetes and the metabolic syndrome: a pathway to cardiovascular disease. Diabetologia 51(4):527-539. https://doi.org/10.1007/ s00125-007-0918-3 
36. Olson TP, Dengel DR, Leon AS, Schmitz KH (2007) Changes in inflammatory biomarkers following one-year of moderate resistance training in overweight women. Int J Obes 31(6):996-1003. https://doi.org/10.1038/sj.ijo.0803534

37. Schumacher A, Peersen K, Sommervoll L, Seljeflot I, Arnesen H, Otterstad JE (2006) Physical performance is associated with markers of vascular inflammation in patients with coronary heart disease. Eur J Cardiovasc Prev Rehabil 13(3):356-362. https://doi. org/10.1097/01.hjr.0000188244.54287.96

38. Adamopoulos S, Parissis J, Kroupis C et al (2001) Physical training reduces peripheral markers of inflammation in patients with chronic heart failure. Eur Heart J 22(9):791-797. https://doi.org/10.1053/ euhj.2000.2285

39. Qiu S, Cai X, Yin H et al (2018) Exercise training and endothelial function in patients with type 2 diabetes: a meta-analysis. Cardiovasc Diabetol 17(1):64. https://doi.org/10.1186/s12933018-0711-2

40. Henson J, Edwardson CL, Bodicoat DH et al (2018) Reallocating sitting time to standing or stepping through isotemporal analysis: associations with markers of chronic low-grade inflammation. J Sports Sci 36(14):1586-1593. https://doi.org/10.1080/02640414. 2017.1405709

41. Phillips CM, Dillon CB, Perry IJ (2017) Does replacing sedentary behaviour with light or moderate to vigorous physical activity modulate inflammatory status in adults? Int J Behav Nutr Phys Act 14(1):138. https://doi.org/10.1186/s12966-017-0594-8
42. Yates T, Khunti K, Wilmot EG et al (2012) Self-reported sitting time and markers of inflammation, insulin resistance, and adiposity. Am J Prev Med 42(1):1-7. https://doi.org/10.1016/j.amepre.2011. 09.022

43. Verdaet D, Dendale P, De Bacquer D, Delanghe J, Block P, De Backer G (2004) Association between leisure time physical activity and markers of chronic inflammation related to coronary heart disease. Atherosclerosis 176(2):303-310. https://doi.org/10.1016/ j.atherosclerosis.2004.05.007

44. Simmons RK, Griffin SJ, Steele R, Wareham NJ, Ekelund U (2008) Increasing overall physical activity and aerobic fitness is associated with improvements in metabolic risk: cohort analysis of the ProActive trial. Diabetologia 51(5):787-794. https://doi.org/10. 1007/s00125-008-0949-4

45. Golubic R, Wijndaele K, Sharp SJ et al (2015) Physical activity, sedentary time and gain in overall and central body fat: 7-year follow-up of the ProActive trial cohort. Int J Obes 39(1):142-148. https://doi.org/10.1038/ijo.2014.66

46. Ekelund U, Brage S, Griffin SJ, Wareham NJ (2009) Objectively measured moderate- and vigorous-intensity physical activity but not sedentary time predicts insulin resistance in high-risk individuals. Diabetes Care 32(6):1081-1086. https://doi.org/10.2337/ dc08-1895

Publisher's note Springer Nature remains neutral with regard to jurisdictional claims in published maps and institutional affiliations. 